\title{
Improving Accuracy of Structural Dynamic Modification with Augmented Residual Vectors
}

\author{
Eric C. Stewart, ${ }^{*}$ \\ NASA, MSFC, AL, 35812 \\ Paul Blelloch, ${ }^{\dagger}$ \\ ATA Engineering Inc., San Diego, CA 92128 \\ Robert N. Coppolino ${ }^{\ddagger}$ \\ Measurement Analysis Coporation Torrance, CA 90505
}

\begin{abstract}
It is often important to perform sensitivity analysis to determine how a structural model will be impacted by design changes. Often, the structural analysts will manually make changes to the finite element model (FEM) to determine the effects. But when dealing with a large FEM with millions of degrees of freedom these manual changes can be cumbersome and calculation of the effects can computationally expensive. Therefore, it is desireable to determine the effects of model changes through approximation methods. One common technique is to determine the analytical sensitivity of the FEM model with respect to the given change. These analytical senstivities are valid when small changes are made to the structural model, but invalid if large changes need to be assessed. Another approach is to use Structural Dynamic Modification (SDM) to create a surrogate model to analyze model changes. SDM is a widely-used sensitivity method and is used in applications of model updating, uncertainty quantification, and model design studies. SMD is valid for moderate (10\%-20\%) changes in the structural model, but model approximations are often needed for large parameter changes $(>\mathbf{2 0 \%})$. Structural Dynamic Modification can be improved by using residual vectors to augment the surrogate model formulation from SDM. Adding the residual modes increases the fidelity of the surrogate model while keeping the computational cost low. This paper discusses the application and limitations of the augmented residual modes method to two structures: the Integrated Spacecraft and Payload Element (ISPE) of the Space Launch System (SLS) and the full SLS as it is configured during its Integrated Modal Test (IMT).
\end{abstract}

\section{Introduction}

After the modal testing of the Space Launch System (SLS) on the Mobile Launcher (ML) the analysts will only have two weeks to perform a model correlation. During this time the NASA engineers will perform a model correlation procedure which parametrically tunes the model to match the test data. This process starts by creating thousands of model perturbations and selecting one that best matches the test data. ${ }^{1}$ However, creating these thousands of perturbations is computationally expensive, and storing the perturbations is yet more expensive due to the size of the model ( $>3$ million degrees of freedom) and density of the modal content in the frequency range of interest $(70$ modes below $7 \mathrm{~Hz})$. One way to reduce the computational time is to use lower-order models to calculate the changes in eigenvectors and eigenvalues from model perturbations.

There are various methods to approximate model changes due to parameter perturbations. One method is to calculate the sensitivities of the eigenvectors and eigenvalues 2 [5 of the model and then use the sensitivities in conjunction with a Taylor series approximation. Another approach is to use Structural Dynamic

*AST, Structural Dynamics, NASA, Marshall Space Flight Center, AL; eric.c.stewart-1@nasa.gov, AIAA Member

$\dagger$ Vice President of Aerospace Analysis, ATA Engineering 13290 Evening Creek Drive, San Diego, CA 92128; paul.blelloch@ata-e.com

${ }^{\ddagger}$ CTO, Measurement Analysis Corporation, 23850 Madison St., Torrance, CA 90505; rcoppolino@macorp.net 
Modification (SDM) to create an approximate model for perturbations 6 The SDM approach has been shown to be more accurate than the Taylor series approximation ${ }^{8]}$ over large parameter changes. However, the SDM approach loses accuracy for large parameter changes (greater than 20\%).

One way to improve the Structural Dynamic Modification method is to append residual modes onto the normal modes of the nominal model.$^{9}$ This increases the fidelity of the model with minimal impact tothe computational cost.

This paper explores the overall accuracy and limitations of adding Augmented Residual Modes to SDM. These accuracy studies will consider both mode frequency and mode shape when large perturbations are made to two large models: the Integrated Stage and Propulsion Element (ISPE) modal test, and the SLS Integrated Modal Test (IMT).

\section{Structural Dynamic Modification and Augmented Residual Modes}

\section{A. Theory}

Structural Dynamic Modification starts with the nominal eigenvalue problem of a structural model

$$
\left[K_{0}\right]\left[\Phi_{0}\right]-\left[M_{0}\right]\left[\Phi_{0}\right]\left[\lambda_{0}\right]=0
$$

The perturbations of the nominal model would take the form

$$
\left[K_{0}+\Delta K\right]\left[\Phi_{2}\right]-\left[M_{0}+\Delta M\right]\left[\Phi_{2}\right]\left[\lambda_{2}\right]=0
$$

where $\Delta K$ and $\Delta M$ are changes in stiffness and mass due to model parameter perturbations. Structural dynamic modification then uses a Ritz series solution to determine the perturbed eigenvalues and eigenvectors. The Ritz series approximation uses the low frequency modes of the nominal system $\Phi_{0 L}$

$$
\left[\Phi_{2}\right]=\left[\Phi_{0 L}\right][\phi]
$$

Using the Ritz series and pre-multiplying by $\Phi_{0}^{T}$ means that Eq. 2 becomes

$$
\left[\Phi_{0 L}\right]^{T}\left[K_{0}+\Delta K\right]\left[\Phi_{0 L}\right][\phi]-\left[\Phi_{0 L}\right]^{T}\left[M_{0}+\Delta M\right]\left[\Phi_{0 L}\right][\phi]\left[\lambda_{2}\right]=0
$$

Which, after distributing the terms, becomes

$$
\left[\left[\Lambda_{0 L}\right]+\left[\Phi_{0 L}\right]^{T}[\Delta K]\left[\Phi_{0 L}\right]\right][\phi]-\left[I+\left[\Phi_{0 L}\right]^{T}[\Delta M]\left[\Phi_{0 L}\right]\right][\phi]\left[\lambda_{2}\right]=0
$$

since

$$
\begin{gathered}
{\left[\Phi_{0 L}\right]^{T}\left[M_{0}\right]\left[\Phi_{0 L}\right]=I} \\
{\left[\Phi_{0 L}\right]^{T}\left[K_{0}\right]\left[\Phi_{0 L}\right]=\Lambda_{O L}}
\end{gathered}
$$

The eigenvalue problem of Eq. 5 is then solved to get a set of eigenvectors and eigenvalues for the perturbed system. Mode shapes of the perturbed system are represented by the physical degrees of freedom as shown in Eq. 3 while the eigenvalues of Eq. 5 are the eigenvalues of the perturbed system.

While SDM is a decent approximation for perturbed mode shapes of a system, the approximation falls apart for large changes $(>20 \%)$ to the model. To improve the SDM approximation, Coppolind ${ }^{9}$ introduced residual vectors. The SDM eigenvalue problem in Eq. 5 becomes

$$
\left[\left[\Lambda_{0 L}\right]+[\bar{\Phi}]^{T}[\Delta K][\bar{\Phi}]\right][\phi]-\left[I+[\bar{\Phi}]^{T}[\Delta M][\bar{\Phi}]\right][\phi]\left[\lambda_{2}\right]=0
$$

The residual vectors are developed by first creating a set of trial modes as

$$
\Psi=\left[\begin{array}{llll}
\Phi_{1 L} & \Phi_{2 L} & \cdots & \Phi_{N L}
\end{array}\right]
$$


where $\Phi_{i L}$ are the low frequency modes of perturbed models. If the nominal model has $N$ parameters that the user wants to use as design variables, then there must be $2 N$ sets of modes to cover the range of variables undergoing large perturbations. The trial vectors are then orthogonalized as

$$
\left[\Psi^{\prime}\right]=\left[I_{O L}-\Phi_{0 L} \Phi_{0 L}^{T} M_{0}\right][\Psi]
$$

However, there can be a large number of trial vectors in $\Psi^{\prime}$, the the number of trial vectors is reduced via singular value decomposition of

$$
\left[\Psi^{\prime T} M \Psi^{\prime}\right][\phi]=[\phi][\lambda]
$$

The trial vectors are reduced by only taking the vectors that have a corresponding singular value above a certain threshold. The variable $\bar{\Phi}$ in Eq. 8 is finally expressed as

$$
[\bar{\Phi}]=\left[\begin{array}{ll}
\Phi_{O L} & \Psi^{\prime} \phi
\end{array}\right]
$$

Calculation of the residual vectors is computationally intensive for models of large structures and with many degrees of freedom. The eigenvalue problem and the singular value decomposition can be very computationally intensive, so a NX NASTRAN DMAP was created to calculate the residual vectors and write them to a .op2 file. The residual vecors are then used in MATLAB to create the approximate models.

\section{B. Implementation}

The calculation of $\Delta K$ and $\Delta M$ from Eq. 2 are determined based on the changes to the design variables in the model. Each parameter in the model $p_{i}$ causes a small change to the mass and stiffness matrices, $\Delta m_{i}$ and $\Delta k_{i}$, respectively. Selection of parameters is important to SDM, as each parameter and its associated mass and stiffness perturbations must have a linear relationship. For a given design, the total change in the mass and stiffness is simply a summation of the changes to the $N$ parameters mulipltied by the small changes to mass and stiffness as

$$
\Delta M=\sum_{i=1}^{N} p_{i} \Delta m_{i} \quad \Delta K=\sum_{i=1}^{N} p_{i} \Delta k_{i}
$$

The parameters used in the current residual vectors method is restricted to be Young's modulii and interface spring stiffnesses because the relationship between $p_{i}$ and the mass and stiffness changes needs to be linear. Selecting Young's modulii and springs stiffnesses also decouples the problem so that now the only the stiffness matrix will change with parameter variations.

The remainder of this paper will focus only on changes to the stiffness matrix $\Delta K$, but an analgous treatment of the mass matrix would apply if material density were chosen as a parameter. To quantify the values of $\Delta k_{i}$, each Young's modulus and interface spring stiffness are independently perturbed to their minimum and maximum limits in the model and the mode shapes and frequencies are calculated. The mode shapes from these model perturbations are the trial modes, $\Phi_{i L}$, from Eq. 9

\section{Models Used}

\section{A. ISPE}

The Integrated Space Payload Element (ISPE) is the part of the Space Launch System (SLS) between the adapters and payloads between the core stage and the Orion crew vehicle as shown in Figure 1 The modal test of the ISPE stack consists of a core stage simulator, the Launch Vehicle Stage Adapter (LVSA), the Interim Cryogenic Propulsion Stage (ICPS), the Orion Multipurpose Crew Vehicle (MPCV) Stage Adapter (OSA), and the MPCV simulator. The model of the ISPE modal test, shown in Figure 2, has over 100 different Young's modulii in the model. To reduce the total number of variables used in the analysis, the different stiffness parameters are grouped by geometric proximity. 


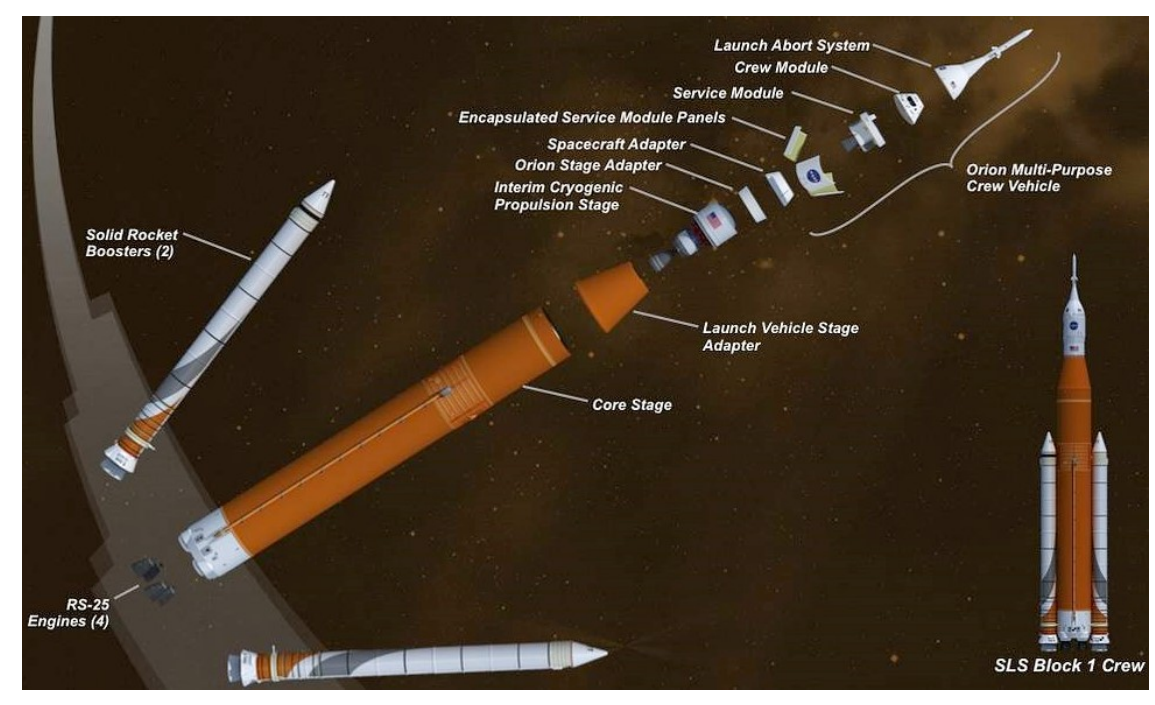

Figure 1. Expanded graphic of the Space Launch System

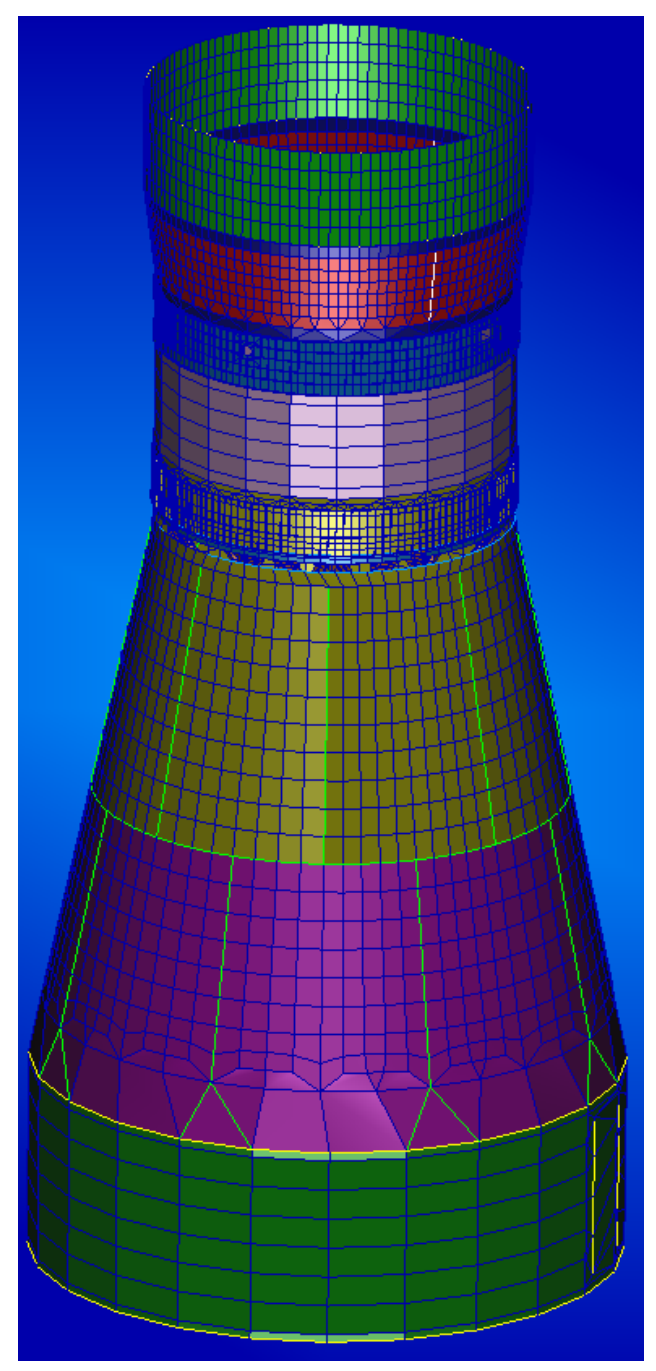

Figure 2. Model of ISPE stack. Grouped parameters are in close proximity to one another and have the same color.

$$
4 \text { of } 6
$$


Variations of $\pm 50 \%$ were applied to each material stiffness parameter (or group of parameters) and $-90 \%$ on the spring stiffnesses, one at a time, to create the trial vectors $\Psi$. As a matter of practical implementation, NX NASTRAN 11.0 has an upper limit on the number of assign statements that can be used in an analysis (approximately 50), so the calculation of the residual vectors had to be split into three different runs. The first run used the trial vectors from all the Youngs modulus $+50 \%$ perturbations and spring stiffnesses $-90 \%$ perturbations. The second run used all of the Youngs' modulus $-50 \%$ perturbations. An SVD cutoff of 1e- 6 was used for each case and modes up to $50 \mathrm{~Hz}$ were calculated. The final NASTRAN residual vector analysis combined the first two residual vector calculations and calcuated modes to $50 \mathrm{~Hz}$ with an SVD cutoff of $1 \mathrm{e}-6$. A total of 847 total residual vectors were kept from the final run.

\section{B. Space Launch System (SLS)}

To test out the effectiveness of the ARM on a full launch vehicle, we used the SLS model in the Integrated Modal Test (IMT) configuration, shown in Figure 3 The SLS launch vehicle is stacked on the Moble Launcher

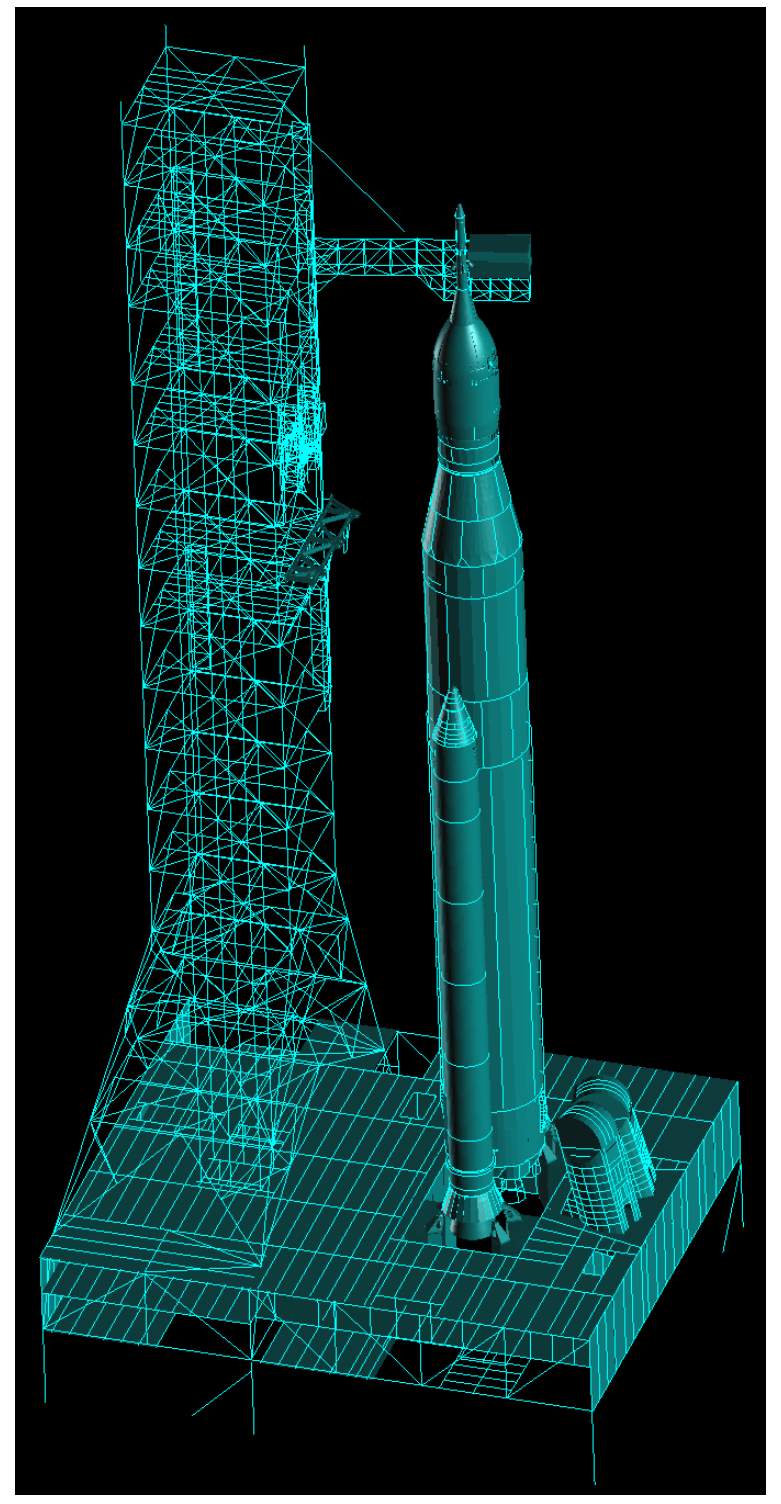

Figure 3. Picture of the SLS in the Integrated Modal Test configuration

(ML) and has the Multi-Purpose Crew Vehicle (MPCV) on top as part of the configuration. Further details 
and results the reduced model improvements due to Augmented Residual Modes will be shown in the final paper.

\section{What's New in this Paper}

This paper explores the overall accuracy and limitations of Augmented Residual Modes and the impact of the residual modes on launch vehicle models. While not shown in this abstract, the accuracy studies are completed for both the ISPE model and the SLS model. The completed paper will show how ARM improves the SDM approximation for both the ISPE and SLS models.

\section{References}

${ }^{1}$ Stewart, E. C. and Hathcock, M. L., "Using Dispersed Modes During Model Correlation," Proceedings of the 2017 AIAA SciTech Forum, 2017.

${ }^{2}$ Fox, R. L. and Kapoor, M. P., "Rates of Change of Eigenvalues and Eigenvectors," AIAA Journal, Vol. 6, No. 12, December 1968 , pp. 2426-2429.

${ }^{3}$ Wang, B., "Improved Approximate MMethod for Computing Eigenvector Derivatives in Structural Dynamics," AIAA Journal, Vol. 29, No. 6, 1990, pp. 1018-1020.

${ }^{4}$ Zhang, O. and Zerva, A., "Iterative MMethod for Calculating Derivatives of Eigenvectors," AIAA Journal, Vol. 34, No. 5, 1996, pp. 1088-1090.

${ }^{5}$ Alvin, K. F., "Efficient Computation of Eigenvector Sensitivities for Structural Dynamics," AIAA Journal, Vol. 35, No. 11, 1997, pp. 1760-1766.

${ }^{6}$ Allemang, R. J., "The Modal Assurance Criterion - Twenty Years of Use and Abuse," Sound and Vibration, August 2003, pp. $14-21$.

${ }^{7}$ Avitabile, P., "Twenty Years of Structural Dynamic Modification - A Review," Sound and Vibration, January 2003, pp. $14-25$.

${ }^{8}$ Yap, K. C. and Zimmerman, D. C., "A Comparitive Study of Structural Dynamic Modification and Sensitivity Method Approximation," Mechanical Systems and Signal Processing, Vol. 16, No. 4, 2002, pp. 585-597.

${ }^{9}$ Coppolino, R. N., FEM Sensitivity Method for Uncertainty and Reconciliation Analyses, Springer New York, New York, NY, 2011, pp. 375-382.

\section{Acknowledgements}

